\title{
EGFR mutation: Significance as a stratification factor in the era of molecular-targeted therapy
}

\author{
YOUNG HAK KIM, KATSUHIRO MASAGO, YOSUKE TOGASHI, YUICHI SAKAMORI, \\ CHIYUKI OKUDA, TADASHI MIO and MICHIAKI MISHIMA
}

Department of Respiratory Medicine, Graduate School of Medicine, Kyoto University, Kyoto 606-8507, Japan

Received November 25, 2010; Accepted January 19, 2011

DOI: $10.3892 / \mathrm{ol} .2011 .240$

\begin{abstract}
Somatic mutations of epidermal growth factor receptor (EGFR) are the strongest predictive markers for the response to EGFR-tyrosine kinase inhibitors (TKIs). Patients with EGFR mutations generally receive EGFR-TKI treatment, and their survival has been significantly improved compared with that before the development of EGFR-TKIs. This study aimed to clarify the impact of EGFR mutational status on the survival of patients with non-small cell lung cancer (NSCLC) receiving cytotoxic agents, but not EGFR-TKIs, as their first-line chemotherapy. In addition, we analyzed patients with EGFR mutations to determine whether the timing of EGFR-TKI administration affects overall survival (OS). A total of 83 NSCLC patients with stage IIIB/IV who received chemotherapy alone and whose EGFR mutational status was known were investigated. Univariate and multivariate analysis for OS was performed using parameters such as age, gender, performance status (PS), histology, disease stage, smoking status, EGFR mutational status and administration of a first-line regimen. Among the 52 patients with EGFR mutations who received EGFR-TKIs, OS between those who received EGFR-TKIs as their first-line treatment and after chemotherapy were similar. Among the 83 patients who received cytotoxic agents as their first-line chemotherapy, the multivariate analysis showed OS to be significantly associated with PS $(\mathrm{p}<0.001)$, histology $(\mathrm{p}=0.039)$ and EGFR mutational status $(\mathrm{p}=0.040)$. OS was almost similar among the 52 patients with EGFR mutations who received EGFR-TKIs in a first- and second-line setting (25.6 vs. 26.8 months, $\mathrm{p}=0.914$ ). The EGFR mutational status had a significant impact on the survival of NSCLC patients, although these patients did not receive EGFR-
\end{abstract}

Correspondence to: Dr Young Hak Kim, Department of Respiratory Medicine, Graduate School of Medicine, Kyoto University, 54 Shogoin-Kawaharacho, Sakyo-ku, Kyoto 606-8507, Japan

E-mail: ekim@kuhp.kyoto-u.ac.jp

Key words: non-small cell lung cancer, chemotherapy, epidermal growth factor receptor, mutation, stratification factor
TKIs as their first-line chemotherapy. In future randomized trials, even when EGFR-TKIs are not included in experimental regimens, patients may need to be stratified by EGFR mutational status in order that study results be evaluated appropriately.

\section{Introduction}

Lung cancer is the leading cause of cancer-related death in many industrialized countries. Platinum-based combination chemotherapy has been shown to improve survival and quality of life in patients with advanced non-small cell lung cancer (NSCLC). However, chemotherapy for advanced NSCLC has been of limited benefit and appears to have reached a plateau, with response rates of approximately $30 \%$ and a median survival period of 8 months (1-4). Various molecular-targeted agents were developed, a number of which are now standard treatment, with or without conventional cytotoxic agents (5-7). Among these agents, tyrosine kinase inhibitors (TKIs) of epidermal growth factor receptor (EGFR) have produced a marked change in the clinical practice of NSCLC.

At present, two different types of EGFR-TKIs are widely used: gefitinib and erlotinib. In predicting the efficacy of these agents, certain clinical factors, such as histology, gender, smoking status and ethnicity, are regarded as significant (8). Somatic mutations of the tyrosine kinase domain of EGFR were found and were shown to be the most reliable predictive marker for the response to EGFR-TKIs (8-10). Findings of a recent population-based study showed that EGFR mutations significantly predict both a survival benefit of gefitinib and a favorable prognosis in patients with advanced lung adenocarcinoma (11). In the recent version of the American Society of Clinical Oncology (ASCO) guideline, gefitinib was accepted as the first-line chemotherapy for patients with activating EGFR mutations (12). The survival benefit is substantial and patients who are known to have EGFR mutations usually receive EGFR-TKIs during the treatment period.

Consequently, the EGFR mutational status may need to be incorporated as a stratification factor in randomized clinical trials even when EGFR-TKIs are not included in the experimental regimens as they appear to strongly affect survival when used in a second-line setting or beyond. This study aimed to show the significance of the EGFR mutational status as a stratification factor for future randomized trials by 
clarifying the impact of the EGFR mutational status on the survival of NSCLC patients receiving cytotoxic agents, but not EGFR-TKIs, as first-line chemotherapy. Additionally, patients with EGFR mutations were examined to determine whether the timing of EGFR-TKI administration plays a role in patient outcome.

\section{Patients and methods}

Patients. Between July 2003 and December 2009, 538 advanced (stage IIIB/IV) NSCLC patients were admitted to our department, and 327 patients received chemotherapy alone. Among them, 116 patients were examined for EGFR mutational status. Of the 116 patients, 83 received cytotoxic agents as their first-line treatment, and the remaining patients received EGFR-TKIs. Of the 116 patients, 52 had activating mutations of EGFR and also received EGFR-TKIs.

This study analyzed the correlation between clinical factors, including EGFR mutational status, evaluated prior to initial treatment, and overall survival (OS) in the 83 patients whose EGFR mutational status was known and who received cytotoxic agents as their first-line treatment (Cohort 1). Among the 52 patients who had EGFR mutations and received EGFRTKIs (Cohort 2), OS was compared between the patients who received EGFR-TKIs as first-line treatment (first-line TKI group; $n=24$ ) and those who received EGFR-TKIs following chemotherapy (second-line TKI group; $\mathrm{n}=28$ ).

Analysis of clinical factors. Analysis of factors such as age ( $<70 / \geq 70$ years), gender (female/male), Eastern Cooperative Oncology Group performance status (PS) (0-1/2-4), histology (adenocarcinoma/non-adenocarcinoma), disease stage (IIIB/ IV), smoking status (+/-), EGFR mutational status (mutation/ wild-type), and administration of a first-line regimen (platinum-based/single-agent) was carried out.

Mutational analysis of EGFR. Formalin-fixed paraffinembedded tissue was cut into 6- to 8-mm sections and mounted on pretreated glass slides. Non-cancer cells and necrotic parts were manually removed from the slide under a microscope. The slides were deparaffinized, and DNA was extracted with phenol-chloroform and ethanol precipitation. The peptide nucleic acid/locked nucleic acid (PNALNA) polymerase chain reaction (PCR) clamp method, designed to detect 11 different EGFR mutations, was used for the determination of the EGFR gene mutation status in this study (13-15).

Tumor evaluation and statistical analysis. Tumor response was assessed according to the Response Evaluation Criteria in Solid Tumors (RECIST) (16). OS was calculated from the commencement of first-line chemotherapy to either the time of death from any cause or the date when patients were last known to be alive. The survival curve was estimated using the Kaplan-Meier method and compared using the log-rank test. Individual clinical factors were compared using the $\chi^{2}$ test. Multivariate analysis was conducted according to the Cox proportional hazards model. $\mathrm{P}<0.05$ was considered to be statistically significant. Statistical analyses were performed using SPSS 11.0 statistical software (SPSS II for Windows, Standard version 11.0; SPSS Inc., Chicago, IL, USA).
Table I. Patient characteristics (Cohort 1).

\begin{tabular}{lc}
\hline Characteristics & $\mathrm{n}=83$ \\
\hline Age (years) & \\
Median & 65 \\
Range & $36-82$ \\
Gender & \\
Female & 39 \\
Male & 44
\end{tabular}

Performance status

$\begin{array}{ll}0-1 & 70\end{array}$

$2-4 \quad 13$

Histology

Adenocarcinoma $\quad 66$

Squamous cell carcinoma $\quad 3$

Large-cell carcinoma $\quad 14$

Disease stage

IIIB 16

IV $\quad 67$

Smoking status

Current smoker $\quad 20$

Former smoker $\quad 25$

Never smoker $\quad 38$

EGFR

Mutation $\quad 28$

Wild-type $\quad 55$

First-line chemotherapy

Platinum-based $\quad 68$

Single-agent $\quad 15$

No. of regimens

Median 3

Range $1-9$

EGFR-TKI treatment

$+\quad 52$

\begin{tabular}{ll}
- & 31 \\
\hline
\end{tabular}

EGFR, epidermal growth factor receptor; TKI, tyrosine kinase inhibitor.

\section{Results}

Cohort 1

Patient characteristics. Table I shows the patient characteristics of Cohort 1 . The median age of the patients was 65 years (range $36-82)$. Of the 83 patients, 39 (47\%) were female, $66(80 \%)$ had histologically confirmed adenocarcinoma and 38 (46\%) were never smokers. Activated EGFR mutations were confirmed in 28 (34\%) patients. A total of $68(82 \%)$ patients received platinumbased regimens and 15 received a single-agent as their first-line chemotherapy. A total of 52 patients $(63 \%)$ received EGFR-TKIs in a second-line setting or beyond. The EGFR mutant patients received EGFR-TKIs (100\%), whereas 24 of 55 wild-type patients received EGFR-TKIs (44\%).

Overall survival. According to the results of the univariate analysis, OS was significantly associated with gender 
Table II. Univariate and multivariate analysis for overall survival (Cohort 1).

\begin{tabular}{|c|c|c|c|c|c|c|}
\hline \multirow[b]{2}{*}{ Characteristics } & \multirow[b]{2}{*}{ No. of patients } & \multirow[b]{2}{*}{ MST (months) } & \multirow{2}{*}{$\begin{array}{c}\begin{array}{c}\text { Univariate } \\
\text { analysis }\end{array} \\
\text { p-value }\end{array}$} & \multicolumn{3}{|c|}{ Multivariate analysis } \\
\hline & & & & Risk ratio & $95 \% \mathrm{CI}$ & $\mathrm{p}$-value \\
\hline \multicolumn{7}{|l|}{ Age } \\
\hline$<70$ & 53 & 23.4 & 0.082 & & & \\
\hline$\geq 70$ & 30 & 10.7 & & & & \\
\hline \multicolumn{7}{|l|}{ Gender } \\
\hline Female & 39 & 25.9 & 0.019 & 1.734 & $0.878-3.427$ & 0.113 \\
\hline Male & 44 & 15.1 & & & & \\
\hline \multicolumn{7}{|l|}{ Performance status } \\
\hline $0-1$ & 70 & 22.5 & $<0.001$ & 3.674 & $1.780-7.581$ & $<0.001$ \\
\hline $2-4$ & 13 & 5.2 & & & & \\
\hline \multicolumn{7}{|l|}{ Histology } \\
\hline Adenocarcinoma & 66 & 23.4 & $<0.001$ & 2.113 & $1.040-4.294$ & 0.039 \\
\hline Non-adenocarcinoma & 17 & 8.1 & & & & \\
\hline \multicolumn{7}{|l|}{ Disease stage } \\
\hline IIIB & 16 & 19.3 & 0.722 & & & \\
\hline IV & 67 & 15.1 & & & & \\
\hline \multicolumn{7}{|l|}{ Smoking status } \\
\hline Current/former & 45 & 13.4 & 0.007 & 0.829 & $0.403-1.706$ & 0.610 \\
\hline Never & 38 & 26.5 & & & & \\
\hline \multicolumn{7}{|l|}{ EGFR } \\
\hline Mutation & 28 & 26.8 & 0.003 & 2.053 & $1.033-4.080$ & 0.040 \\
\hline Wild-type & 55 & 10.6 & & & & \\
\hline \multicolumn{7}{|l|}{ First-line regimen } \\
\hline Platinum-based & 68 & 20.1 & 0.082 & & & \\
\hline Single-agent & 15 & 10.3 & & & & \\
\hline
\end{tabular}

MST, median survival time; EGFR, epidermal growth factor receptor.
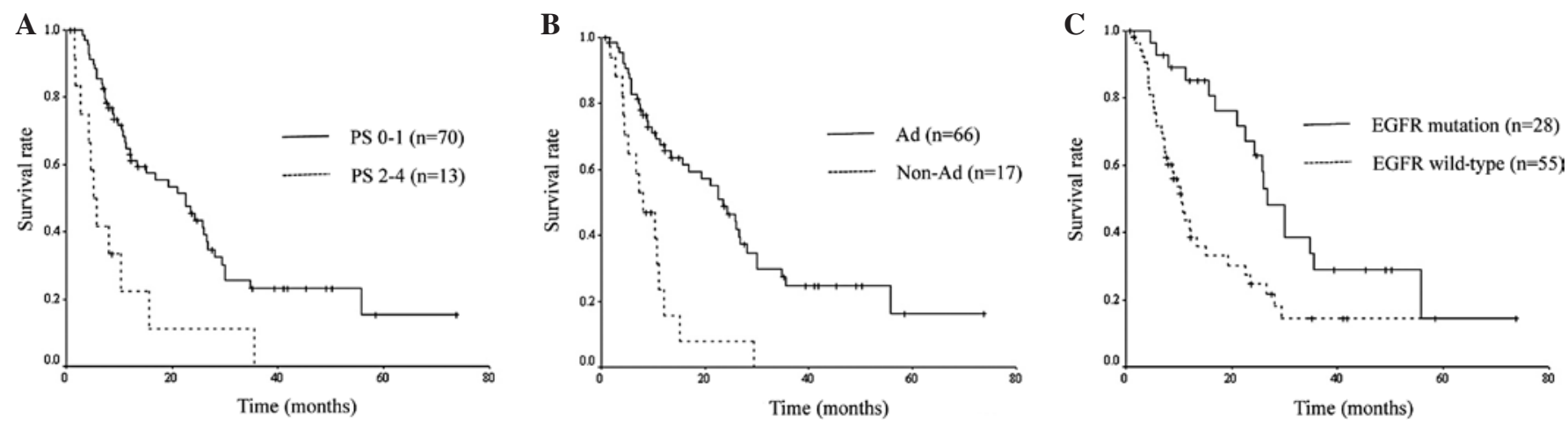

Figure 1. Overall survival curves according to (A) performance status (PS), (B) histology and (C) EGFR mutational status. Ad, adenocarcinoma.

$(\mathrm{p}=0.019)$, PS $(\mathrm{p} \leq 0.001)$, histology $(\mathrm{p}<0.001)$, smoking status $(\mathrm{p}=0.007)$ and EGFR mutational status $(\mathrm{p}=0.003)$. Multivariate analysis identified PS $(\mathrm{p}<0.001)$, histology $(\mathrm{p}=0.039)$ and EGFR mutational status $(\mathrm{p}=0.040)$ as independent prognostic factors for OS (Table II). Survival curves drawn according to PS, histology and EGFR mutational status are shown in Fig. 1.

\section{Cohort 2}

Patient characteristics. Table III shows the patient characteristics of Cohort 2. Compared to the second-line TKI group, the first-line TKI group comprised more elderly and more PS 2-4 patients, whereas the proportion of females was higher in the first-line TKI group. All patients had histologicaly confirmed adenocarcinoma. 
Table III. Patient characteristics (Cohort 2).

\begin{tabular}{lccc}
\hline Characteristics & First-line TKI group $(\mathrm{n}=24)$ & Second-line TKI group $(\mathrm{n}=28)$ & $\mathrm{p}$-value \\
\hline Age (years) & 74 & 61 & 0.001 \\
Median & $34-86$ & $39-74$ & \\
Range & 20 & 15 & 0.023 \\
Gender & 4 & 13 & 0.104 \\
Female & & & \\
Male & 16 & 24 & - \\
Performance status & 8 & 4 & 0.910 \\
$0-1$ & & 28 & \\
$2-4$ & 24 & 0 & \\
Histology & 0 & 5 & \\
Adenocarcinoma & & 23 & \\
Non-adenocarcinoma & 4 & & \\
Disease stage & 20 & & \\
IIIB & & & \\
IV & & & \\
\hline
\end{tabular}

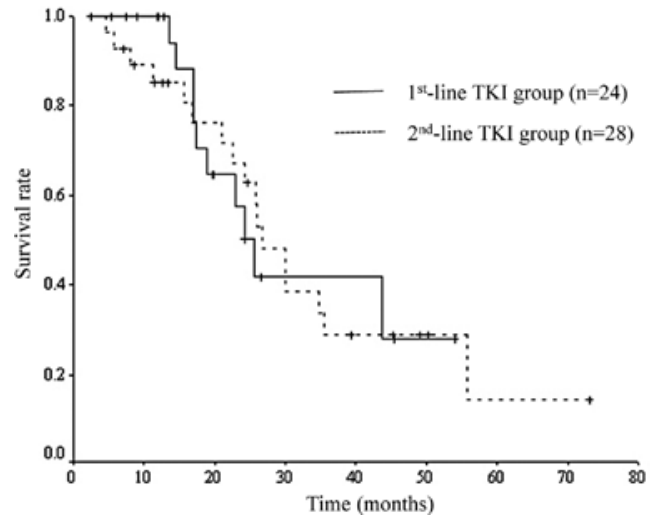

Figure 2. Overall survival curves according to the timing of EGFR-TKI administration in patients with EGFR mutations.

Overall survival. OS of the first-line TKI group was 25.6 months and that of the second-line TKI group was 26.8 months. No significant difference was noted between the two groups $(p=0.914)$. Survival curves of the two groups are shown in Fig. 2.

\section{Discussion}

Recently, effective second-line chemotherapies have been developed using agents, such as docetaxel, pemetrexed, gefitinb and erlotinib, for the treatment of NSCLC (12). Therefore, OS is not necessarily the most favorable primary endpoint in randomized trials since the exact difference of effectiveness between the investigated regimens does not often translate into OS due to effective second-line chemotherapy or beyond. Consequently, progression-free survival has been selected as a primary endpoint in a number of recent randomized trials. However, progression-free survival is less reliable than OS due to its arbitrariness, and it remains debatable which is more adequate as a primary endpoint (17-19). Should a definite biomarker be found that predicts the response of second-line chemotherapy and patients are stratified based on such a biomarker, then OS is likely to be the most favorable endpoint.

The development of EGFR-TKIs and the finding of EGFR mutations is a significant event in chemotherapy for NSCLC, since individualized therapy is now feasible (20). Currently, the EGFR mutation is the most powerful and widespread biomarker in NSCLC. A number of biomarkers, such as excision repair cross-complementation group 1 (ERCC1)21 for cisplatin, ribonucleotide reductase subunit M1 (RRM1)22 for gemcitabine and thymidylate synthase (TS)23 for pemetrexed, have been identified. However, no other biomarker apart from the EGFR mutation has been of clinical use.

In the present study, the correlation between clinical factors, including the EGFR mutational status evaluated prior to the initial treatment, and OS was analyzed. We found that the EGFR mutational status was an independent prognostic factor for OS as well as PS and histology in NSCLC patients who received cytotoxic agents, but not EGFR-TKIs, as their first-line treatment. In addition, the efficacy of EGFR-TKIs was similar regardless of the timing of the administration when the patients had EGFR mutations, as previously reported (24). Patients who are known to have EGFR mutations are generally treated with EGFR-TKIs; in the present study, any patients who had EGFR mutations were treated with EGFR-TKIs.

The prevalence of EGFR mutations is much higher in Asia than in Western populations (8). Therefore, the importance of EGFR mutations as a stratification factor is prominent in Asian-oriented trials, although the same may not be the case of Western-oriented trials. Our results should be confirmed in non-Asian regions as well. More globalized clinical trials are currently underway and the role of EGFR mutations as a stratification factor appears to be of significance in such global trials.

In conclusion, the EGFR mutational status was an independent prognostic factor for survival in NSCLC patients who received cytotoxic agents, but not EGFR-TKIs, as their first-line chemotherapy. In future randomized trials, particularly in Asia, even when EGFR-TKIs are not included in the 
experimental regimens, patients may need to be stratified by EGFR mutational status to for study results to be evaluated appropriately.

\section{References}

1. Spiro SG and Porter JC: Lung cancer - where are we today? Current advances in staging and nonsurgical treatment. Am J Respir Crit Care Med 166: 1166-1196, 2002.

2. Kelly K, Crowley J, Bunn PA Jr, et al: Randomized phase III trial of paclitaxel plus carboplatin versus vinorelbine plus cisplatin in the treatment of patients with advanced non-small cell lung cancer: a Southwest Oncology Group trial. J Clin Oncol 19 : 3210-3218, 2001.

3. Schiller JH, Harrington D, Belani CP, et al: Comparison of four chemotherapy regimens for advanced non-small cell lung cancer. N Engl J Med 346: 92-98, 2002.

4. Ohe Y, Ohashi Y, Kubota K, et al: Randomized phase III study of cisplatin plus irinotecan versus carboplatin plus paclitaxel, cisplatin plus gemcitabine, and cisplatin plus vinorelbine for advanced non-small-cell lung cancer: Four-Arm Cooperative Study in Japan. Ann Oncol 18: 317-323, 2007.

5. Shepherd FA, Rodrigues-Pereira J, Ciuleanu T, et al: Erlotinib in previously treated non-small-cell lung cancer. N Engl J Med 353: 123-132, 2005.

6. Sandler A, Gray R, Perry MC, et al: Paclitaxel-carboplatin alone or with bevacizumab for non-small-cell lung cancer. N Engl J Med 355: 2542-2550, 2006.

7. Kim ES, Hirsh V, Mok T, et al: Gefitinib versus docetaxel in previously treated non-small-cell lung cancer (INTEREST): a randomised phase III trial. Lancet 372: 1809-1818, 2008.

8. Mitsudomi T and Yatabe Y: Mutations of the epidermal growth factor receptor gene and related genes as determinants of epidermal growth factor receptor tyrosine kinase inhibitors sensitivity in lung cancer. Cancer Sci 98: 1817-1824, 2007.

9. Lynch TJ, Bell DW, Sordella R, et al: Activating mutations in the epidermal growth factor receptor underlying responsiveness of non-small-cell lung cancer to gefitinib. N Engl J Med 350: 2129-2139, 2004.

10. Paez JG, Janne PA, Lee JC, et al: EGFR mutations in lung cancer: correlation with clinical response to gefitinib therapy. Science 304: 1497-1500, 2004.

11. Takano T, Fukui T, Ohe Y, et al: EGFR mutations predict survival benefit from gefitinib in patients with advanced lung adenocarcinoma: a historical comparison of patients treated before and after gefitinib approval in Japan. J Clin Oncol 26: 5589-5595, 2008.

12. Azzoli CG, Baker S Jr, Temin S, et al: American Society of Clinical Oncology Clinical Practice Guideline update on chemotherapy for stage IV non-small-cell lung cancer. J Clin Oncol 27: 6251-6266, 2009.
13. Nagai Y, Miyazawa H, Huqun, et al: Genetic heterogeneity of the epidermal growth factor receptor in non-small cell lung cancer cell lines revealed by a rapid and sensitive detection system, the peptide nucleic acid-locked nucleic acid PCR clamp. Cancer Res 65: 7276-7282, 2005.

14. Sutani A, Nagai Y, Udagawa K, et al: Gefitinib for non-smallcell lung cancer patients with epidermal growth factor receptor gene mutations screened by peptide nucleic acid-locked nucleic acid PCR clamp. Br J Cancer 95: 1483-1489, 2006.

15. Tanaka T, Nagai Y, Miyazawa H, et al: Reliability of the peptide nucleic acid-locked nucleic acid polymerase chain reaction clamp-based test for epidermal growth factor receptor mutations integrated into the clinical practice for non-small cell lung cancers. Cancer Sci 98: 246-252, 2007.

16. Therasse P, Arbuck SG, Eisenhauer EA, et al: New guidelines to evaluate the response to treatment in solid tumors. European Organization for Research and Treatment of Cancer, National Cancer Institute of the United States, National Cancer Institute of Canada. J Natl Cancer Inst 92: 205-216, 2000.

17. Buyse ME, Squifflet P, Laporte S, et al: Prediction of survival benefits from progression-free survival in patients with advanced non-small cell lung cancer: evidence from a pooled analysis of 2,838 patients randomized in 7 trials. ASCO Meeting Abstracts 26 (Suppl 15): abs. 8019, 2008.

18. Hotta K, Kiura K, Takigawa N, et al: Progression-free survival (PFS) and overall survival (OS) in phase III trials of systemic chemotherapy in advanced non-small cell lung cancer (NSCLC). ASCO Meeting Abstracts 28 (Suppl 15): abs. 7624, 2010.

19. Malik SM, Ibrahim A, Sridhara R, et al: Use of progressionfree survival (PFS) as an endpoint in advanced non-small cell lung cancer (NSCLC) trials: FDA perspective. ASCO Meeting Abstracts 28 (Suppl 15): e18001, 2010.

20. Mok TS, Wu YL, Thongprasert S, et al: Gefitinib or carboplatinpaclitaxel in pulmonary adenocarcinoma. N Engl J Med 361: 947-957, 2009.

21. Olaussen KA, Dunant A, Fouret P, et al: DNA repair by ERCC1 in non-small-cell lung cancer and cisplatin-based adjuvant chemotherapy. N Engl J Med 355: 983-991, 2006.

22. Souglakos J, Boukovinas I, Taron M, et al: Ribonucleotide reductase subunits M1 and M2 mRNA expression levels and clinical outcome of lung adenocarcinoma patients treated with docetaxel/gemcitabine. Br J Cancer 98: 1710-1715, 2008.

23. Ceppi P, Volante M, Saviozzi S, et al: Squamous cell carcinoma of the lung compared with other histotypes shows higher messenger RNA and protein levels for thymidylate synthase. Cancer 107: 1589-1596, 2006.

24. Wu JY, Yu CJ, Yang CH, et al: First- or second-line therapy with gefitinib produces equal survival in non-small cell lung cancer. Am J Respir Crit Care Med 178: 847-853, 2008. 